\title{
Estudo sobre Publicações em Mensuração e Avaliação de Desempenho em Revistas de Contabilidade da Espanha
}

\author{
Study of Publications in Performance Measurement and Evaluation in Spain \\ Accounting Journals \\ Rogério João Lunkes \\ rogeriolunkes@hotmail.com \\ UFSC
}

\author{
Vicente Mateo Ripoll \\ Vicente.Ripoll@uv.es \\ UFSC
}

\author{
Fabricia Silva da Rosa \\ fabriciasrosa@hotmail.com \\ UFSC
}

\begin{abstract}
Resumo
A mensuração e avaliação de desempenho é um dos temas de grande discussão na literatura, principalmente na contabilidade gerencial. Uma das formas de perceber e entender o seu nível de desenvolvimento é estudar suas publicações nas principais revistas. Assim, o objetivo do trabalho é identificar e analisar as publicações sobre mensuração e avaliação de desempenho nas revistas de contabilidade da Espanha. Para tanto, foram selecionadas sete revistas espanholas listadas no IN-RECS, e analisados 55 artigos no período de 2001 a 2010. Entre os principais resultados pode-se destacar a abordagem do tema diretamente relacionado á aplicação do Cuadro de Mando Integral, com utilização dos métodos predominantemente de revisão e estudo de caso. Já entre as citações, forte influencia da literatura norte-americana.
\end{abstract}

Palavras-chave: Mensuração, avaliação, desempenho, balanced scorecard.

\begin{abstract}
The performance measurement and evaluation is a topic of great discussion in the literature, mostly in managerial accounting. One way to perceive and understand their level of development is to study his publications in major journals. Thus, the objective is to identify and analyze the publications in performance measurement and evaluation in Spain accounting journals. To this end, we selected seven Spanish journals listed in the IN-RECS, and analyzed 55 articles from 2001 to 2010 . The main results can be highlighted to approach the subject directly related to the implementation of the Balanced Scorecard, using mainly the methods of review and case study. Among the citations, strong influences of American literature.
\end{abstract}

Keywords: Performance, measurement, evaluation, Balanced Scorecard.

Artigo recebido em: 06.07.2012; Aceito em: 11.12.2012

\section{INTRODUÇÃO}

A gestão das organizações sempre foi uma atividade complexa, com influência de múltiplas variáveis e sujeita a riscos. Entretanto, nas últimas décadas, face à abertura dos mercados, internacionalização e consequente volatilidade do capital, crises financeiras e econômicas e aos avanços tecnológicos, essa complexidade teve um aumento significativo.

Em resposta a essas mudanças, as organizações necessitam identificar e gerenciar os fatoreschave de seu sucesso. Aliado a isto, necessitam de sistemas de mensuração e avaliação de 
desempenho, visando estabelecer suas linhas de ação e verificar seu nível de competitividade. A mensuração e avaliação de desempenho é um processo de registro, acumulação, disseminação e utilização da informação e estruturação das medidas de gestão, visando medir e avaliar o grau de alcance dos objetivos e metas.

Assim como na prática, na literatura o tema mensuração e avaliação de desempenho também é amplamente explorado (Johnson e Kaplan, 1987; Cooper, 1987; Kaplan, 1983; Hiromoto, 1988; Bromwich, 1990; Dent, 1990; Young e Selton, 1991; Kaplan e Norton, 1992; Shank e Govindarajan, 1993; Simons, 1995 e Atkinson, 2000), inclusive com aplicação de estudos com perspectivas multidisciplinares (Baiman, 1982; Cooper, 1983; Covaleski, Dirsmith, e Samuel, 1996; Hopwood, 1978; Macintosh e Scapens, 1990; Kaplan e Norton, 2001).

Apesar deste grande sucesso, principalmente pela aplicação de ferramentas como o Cuadro de Mando Integral ou Balanced Scorecard, tem surgido criticas de que os trabalhos gerados são limitados e não trazem soluções que possam ser generalizadas, ou seja, possam se tornar teorias. Zimmerman (2001) aponta que o estágio da produção científica da contabilidade gerencial de uma forma geral vem restringindo-se a estudos de casos, e que não tem possibilidade de se tornar uma teoria generalizada, bem como valoriza trabalhos práticos, que não estão proporcionando base sólida para o crescimento do conhecimento. Ittner e Larcker (2001) mencionam que a generalização de conhecimentos no sentido de criar e solidificar abordagens teóricas mais consistentes não tem ocorrido com tanta frequência. Entre as causas, destacam que muitos tópicos não são amadurecidos criticamente e aprofundados ao longo do tempo, gerando lacunas teóricas não resolvidas, isto porque com frequência não há continuidade dos estudos.

Entre os temas que tem recebido criticas pela limitação de seus estudos, é mensuração e avaliação de desempenho, principalmente aqueles relacionados com a aplicação do CMI ou BSC. Portanto, estudar como esse tema é abordado na literatura, principalmente em revistas, é importante para conhecer seu nível de desenvolvimento e inovação, além de possíveis limitações. Isto pode proporcionar informações importantes para estudiosos do tema e auxiliar na melhoria das aplicações práticas nas organizações. Para tanto, o objetivo do trabalho é identificar e analisar os trabalhos de mensuração e avaliação de desempenho nas publicações em revistas espanholas de contabilidade.

A justificativa do estudo está na percepção de que o desenvolvimento das ciências depende de estudos capazes de produzir um conjunto substantivo de conhecimento, mas também de trabalhos que se dediquem à análise e reflexão crítica sobre a produção cientifica gerada.

\section{MENSURAÇÃO E AVALIAÇÃO DE DESEMPENHO - MAD}

A avaliação de desempenho é um processo para analisar problemas complexos, que envolve múltiplos critérios, fatores subjetivos e objetivos a serem considerados no processo decisório (Ensslin et al., 2011), e tem o intuito de fornecer aos gestores uma base racional para tomada de decisões (Mohamadabi, Tichkowsku e Kumar, 2009 ou para construir conhecimento no decisor e permitir que o mesmo conheça a priori as consequiências de suas decisões (Ensslin et al., 2011), para analisar documentos (Aliguliyev, 2009), processos (Balasubramanian e Gupta, 2005) e para promover a aprendizagem contínua (Chin, Lo e Leung, 2010).

Em uma visão prescritivista a avaliação de desempenho é vista como um processo para gerar conhecimento organizacional a partir do conhecimento tático de especialistas (Abernethy et $a l .$, 2005). Ou a partir da percepção dos analistas com a finalidade de sugerir ou prescrever como os gestores devem tomar decisões, e também, possibilitar geração e comparação entre alternativas para solucionar o problema avaliado (Hsu, Li e Chen, 2009), e estão apoiadas em critérios e sub-critérios de análise identificados por especialistas (Li e Liao, 2007). 
Em uma visão construtivista (Enssin et al., 2010; Lacerda, Ensslin e Ensslin, 2010) é um instrumento utilizado para construir o entendimento de formas a permitir pró-ativamente conhecer as consequências das decisões e ou alternativas, segundo a percepção dos gestores.

A avaliação de desempenho requer um processo estruturado (Ensslin et al, 2010, Lacerda, Ensslin e Ensslin, 2010, Rosa, Ensslin e Ensslin, 2009), ou um conjunto de critérios estabelecidos pelos especialistas (Barber e Scarcelli, 2010; Chen, Huang e Cheng, 2009), ou ainda um conjunto de critérios pré-estabelecidos na literatura. Alguns dos modelos resultam também em um conjunto de alternativas reais que podem se alterar durante a sua construção, e assim servem também para gerar novas alternativas (Hu, Lee, Yen e Tsai, 2009; Janssen et al, 2005; Selden e Sowa, 2004; Wouters e Wilderon, 2008; Carl e Chris, 2003).

Observa-se também diferentes objetivos para as ferramentas de avaliação de desempenho, assim podem auxiliar na tomada de decisão a respeito da escolha de alternativas (Barber e Scarcelli, 2010; Chen, Huang e Cheng, 2009) ou para apoiar decisões a partir da percepção daquele que sofre com as conseqüências da decisão (Ensslin et al., 2010; Lacerda, Ensslin e Ensslin, 2011, Rosa, Ensslin e Ensslin, 2010).

Também é utilizada para analisar as relações entre as variáveis e estabelecer como os gestores decidem na prática, para mensurar aspectos financeiros e não financeiros a partir do mapa estratégico da empresa (Azofra, Prieto e Alicia, 2003), e para construir cenários futuros baseados em mapas estratégicos, considerando as preocupações dos stakeholders (Buytendijk, Hatch e Pietro, 2010).

Os aspectos relevantes em um modelo de avaliação de desempenho, segundo Keeney (1992), podem ser o objetivo, critério, atributo, fator crítico de sucesso ou ponto de vista fundamental. Para identificar esses critérios é possível basear-se no conhecimento dos decisores, de critérios pré-estabecidos na literatura e normas, ou o conhecimento de especialistas.

Assim, para Abernethy, Home, Lillis, Malina e Selto (2005); Buytendijk, Hatch e Pietro (2010; Selden e Sowa (2004); Wouters e Wilderon (2008) os critérios podem ser identificados por meio de entrevistas e questionário. Já Aliguliyev (2009) identifica os critérios utilizando software que organiza em clusters critérios com características semelhantes, em documentos internos da empresa analisada. Já para Azofra, Prieto e Alicia (2003); Balasubramanian e Gupta (2005); Barber e Scarcelli (2010); Carl e Chris (2003); Chen, Huang, Cheng (2009); Hsu, Li e Chen (2010) e Hu, Lee, Yen e Tsai (2009) os critérios e sub-critérios são identificados na literatura. Chin, Lo e Leung (2010); Dey (2006); Li e Liao (2007) e Kumar (2008) baseiam-se no conhecimento de especialistas; Carl e Chris (2003) identificam os critérios na estratégia da organização estudada, e Ensslin et al. (2010); Lacerda, Ensslin e Ensslin (2011); Rosa, Ensslin e Ensslin (2010) o modelo é construído a partir da percepção do decisor.

Os modelos de avaliação de desempenho possibilitam também mensurar os critérios identificados. Esta mensuração pode ser feita por meio de escalas do tipo Likert (Azofra, Peitro e Alcia, 2003; Carl e Chris, 2003; Chen, Huang e Cheng, 2009; Chin e Lo, e Leng, 2010; Hu, Lee, Yen e Tsai, 2009; Janssena, Goosena, Verhoevenb, Verhoevenb, Omtzigta e Matby, 2005; e Li e Lao, 2007). Por atribuição de pesos ou médias ponderadas Aliguliyev (2009); Balasubramanian e Gupta (2005); Barber e Scarcelli (2010); Selden e Sowa (2004); e Wouters e Wilderon (2008). Sendo que a integração dos critérios mensurados por essas escalas pode ser realizada por tabulação ou ponderação, (Azofra, Peitro e Alcia, 2003; Carl e Chris, 2003; Chen, Huang e Cheng, 2009; Chin e Lo, e Leng, 2010; Hu, Lee, Yen e Tsai, 2009; Janssena, Goosena, Verhoevenb, Verhoevenb, Omtzigta e Matby, 2005; e Li e Lao, 2007), desta forma, os resultados apresentados são tão confiáveis quanto indicar a precisão estatística, também se pode entender que os resultados representam a verdade, ou seja, são normas de comportamento racional, assim, consideram que os resultados são verdades para o 
decisor racional, além disso, os resultados são vistos como prescrições, que devem ser seguidos pelos decisores, para manter a sua coerência lógica.

Também pode ser feita por descritores que medem o grau com que um objetivo é alcançado, e que apresentam as seguintes propriedades: mensurável, operacional, inteligível, homogêneo, que permite distinguir o desempenho melhor e pior, e respeita as propriedades das escalas ordinais (Keeney, 1992 e Ensslin et al., 2010). A integração dos descritores pode ser feita a partir de funções de valor que permitem a avaliação local e para avaliação global, as denominadas taxas de substituição que expressam, segundo o julgamento dos decisores, a perda de performance que uma ação potencial deva sofrer em um critério para compensar o ganho de desempenho em outro (ENSSLIN, MONTIBELLER e NORONHA, 2001). Assim, os resultados permitem identificar o perfil de desempenho das ações e visualizar gráfica e numericamente as consequências das ações nos aspectos julgados pelo decisor como estratégicos para a avaliação do contexto.

Em razão do objetivo do trabalho apenas serão mencionados, entre os inúmeros diferentes modelos e sistemas de mensuração e avaliação de desempenho, para fins de análise o Cuadro de Mando e o Cuadro de Mando Integral.

Utilizado a varias décadas (Blanco, 1976), o Cuadro de Mando (CM), que tem sua origem no Tableau de Bord proposto por engenheiros franceses nos anos sessenta (Meyer, 1969), é um sistema de medição que pretende visualizar a informação útil para diagnosticar a situação e poder gerenciar a empresa (Fernández, 2000; Ballvé e Amat Salas, 2006).

Por sua vez, o Cuadro de Mando Integral (CMI) ou Balanced Scorecard se introduziu pela primeira vez a princípios dos anos noventa (Kaplan e Norton, 1992). Desde então, muitas organizações do mundo tem implementado, isto porque o consideram uma ferramenta de gestão útil, que lhes permite definir seus próprios modelos ou mapas de negócios com uma visão ampla da organização, facilitando a comunicação e implementação da estratégia.

\section{METODOLOGIA DE PESQUISA}

Nesta seção apresentam-se os procedimentos metodológicos utilizados para a construção do referencial teórico, em seguida o processo de seleção dos artigos, além da sua classificação quanto ao tema. Esta seleção ocorreu em duas etapas, à primeira com a seleção das revistas e classificação dos artigos em temas, e a segunda com a estratificação do tema mensuração e avaliação de desempenho (MAD), com a sua classificação e a analise das suas principais características.

\subsection{Processo estruturado para construção do referencial teórico}

O processo estruturado para seleção e análise de referências bibliográficas está dividido em três fases: escolha das bases de dados, seleção de artigos e análise sistêmica (Rosa, Ensslin e Ensslin, 2009). A primeira fase fundamenta a escolha de base de dados; a segunda obtém um portfólio de artigos a partir de processo estruturado e fundamentado, e por fim, tem-se a terceira fase para proporcionar a análise sistêmica do portfólio de artigos.

Com base nesta estrutura foram selecionados para análise sistêmica do referencial teórico 26 artigos alinhados com o tema da pesquisa "MAD", para alcançar esta quantidade de artigos, as fases e etapas foram feitas de forma sequencial. 


\subsection{Seleção dos Artigos}

Este estudo tem como objetivo pesquisar somente as publicações em revistas de contabilidade editadas na Espanha. Para tanto, são consideradas algumas variáveis, tais como: (i) este estudo esta centrado unicamente em contabilidade gerencial, (ii) para seleção das revistas considerou-se o termo "contabilidad" ou "contable" e sua inclusão no IN-RECS (Índice de impacto das revistas espanholas de ciências sociais, elaborado pela Universidade de Granada; (iii) para seleção dos artigos foi considerado um período de 10 anos (2001 a 2010). A escolha do IN-RECS deve-se a sua grande utilização na Espanha, além da impossibilidade de outra base de dados, já que o país possui apenas uma revista de contabilidade com indexação mundial (como por exemplo no ISI - International Statistical Institute ou JCR - Journal Citation Reports). A Revista Española de Financiación y Contabilidad é a única indexada no JCR.

Conforme Tabela 1, a partir das sete revistas foram selecionados 421 artigos relacionados em contabilidade gerencial. Esta seleção foi realizada manualmente com a leitura do resumo e, quando necessária, a análise do artigo na integra.

Os resultados mostram que aproximadamente $11 \%$ dos artigos publicados durante o período estão relacionados com contabilidade gerencial. Entretanto, se excluída da amostra a revista RICG, exclusiva em contabilidade gerencial, esta porcentagem se reduz a aproximadamente 8\%, o que indica um baixo nível de publicação nesse campo na Espanha, principalmente se compararmos as revistas norte-americanas de contabilidade que apresentam $28 \%$ das publicações em contabilidade gerencial (Hesford et al., 2007).

Tabela 1 Resultados das revistas selecionadas

\begin{tabular}{|c|c|c|c|c|}
\hline $\begin{array}{c}\text { Posição } \\
\text { Período } \\
\text { (2001- } \\
\text { 2009) }\end{array}$ & Revistas & $\begin{array}{l}\text { Citas por } \\
\text { artículo }\end{array}$ & $\begin{array}{c}\text { Total de } \\
\text { artigos } \\
(2001 \text { a 2010) }\end{array}$ & $\begin{array}{c}\text { Artigos } \\
\text { Selecionados } \\
(2001 \text { a 2010) }\end{array}$ \\
\hline 15 & $\begin{array}{l}\text { Revista Española de Financiación y Contabilidad } \\
\text { - REFC }\end{array}$ & 0.810 & 332 & $40(12 \%)$ \\
\hline 19 & Revista de Contabilidad - RC & 0.688 & 117 & $15(13 \%)$ \\
\hline 44 & Revista de Contabilidad y Tributación - RCT & 0.310 & 764 & $18(2,5 \%)$ \\
\hline 77 & $\begin{array}{l}\text { Partida Doble Revista de Contabilidad, Auditoría } \\
\text { y Empresa - RCAE }\end{array}$ & 0.087 & 921 & $89(9,5 \%)$ \\
\hline 88 & Técnica Contable - RTC & 0.051 & 612 & $44(7 \%)$ \\
\hline 99 & $\begin{array}{l}\text { Revista Iberoamericana de Contabilidad de } \\
\text { Gestión - RICG }\end{array}$ & 0.037 & $125^{*}$ & $125(100 \%)$ \\
\hline 108 & $\begin{array}{l}\text { Harvard-Deusto Finanzas \& Contabilidad - } \\
\text { RHDFC }\end{array}$ & 0.024 & $449 * *$ & $90(20 \%)$ \\
\hline Total & & - & 3320 & $421(11 \%)$ \\
\hline
\end{tabular}

Fonte: Própria

* Disponível a partir de 2003. ** Publicação de uma edição da revista em 2001.

Os resultados também mostram que não houve um crescimento nas publicações em contabilidade gerencial mesmo com a criação de uma revista exclusiva, ao contrario está 
ocorrendo uma diminuição no número de artigos publicados (Ver Gráfico 1). A média de publicação anual em contabilidade gerencial é de aproximadamente 42 artigos. Isto mostra que ao contrario de outros países, como por exemplo, os EUA, onde houve um aumento em termos absolutos e uma diminuição em termos relativos (Hesford et al., 2007), na Espanha as publicações em contabilidade gerencial principalmente nos últimos anos tem uma diminuição, tanto em termos relativos como absolutos.

Visando obter a amostra da pesquisa, na seqüência os artigos são classificados em temas.

\subsection{Classificação dos Artigos}

Como ponto de partida para a classificação dos artigos de contabilidade gerencial é utilizada a tradicional divisão já aceita na literatura, entre "custos" e "planejamento e controle", além dos temas específicos denominado de "outros". A divisão dos temas principais em subdivisões levou em consideração os estudos realizados por Schekaiban e Ripoll (2005), Hesford et al. (2007) e Lunkes, Ripoll e Rosa (2011) e esta apresentada na Tabela 2.

Tabela 2 Resultados da classificação dos temas

\begin{tabular}{|c|c|c|c|c|c|c|c|c|}
\hline Temas & REFC & $\mathbf{R C}$ & RCT & RCAE & RTC & RICG & RHDFC & Total \\
\hline \multicolumn{9}{|l|}{ Custos } \\
\hline Alocação de custos & $2(5 \%)$ & $2(13 \%)$ & $1(6 \%)$ & $12(14 \%)$ & $10(23 \%)$ & $9(7 \%)$ & $7(8 \%)$ & $43(10,5 \%)$ \\
\hline $\begin{array}{l}\text { Outros tópicos de contabilidade } \\
\text { de custos }\end{array}$ & & & & $1(1 \%)$ & $3(7 \%)$ & $1(1 \%)$ & $2(2 \%)$ & $7(1,5 \%)$ \\
\hline Estudos e Praticas de custos & $1(2,5 \%)$ & & & $4(4,5 \%)$ & $3(7 \%)$ & $13(10 \%)$ & $6(7 \%)$ & $27(6,5 \%)$ \\
\hline $\begin{array}{l}\text { ABM(Administração Baseada } \\
\text { em Atividades) }\end{array}$ & & & & $9(10 \%)$ & $1(2 \%)$ & $2(2 \%)$ & $2(2 \%)$ & $14(3,5 \%)$ \\
\hline Múltiplos & $1(2,5 \%)$ & & & $5(5,5 \%)$ & & $7(6 \%)$ & $4(4 \%)$ & $17(4 \%)$ \\
\hline Sub-total & $4(10 \%)$ & $2(13 \%)$ & $1(6 \%)$ & $31(35 \%)$ & $17(39 \%)$ & $32(26 \%)$ & $21(23 \%)$ & $108(26 \%)$ \\
\hline \multicolumn{9}{|l|}{ Planejamento e controle } \\
\hline Orçamento & $2(5 \%)$ & & & $5(5,5 \%)$ & $2(4,5 \%)$ & $5(4 \%)$ & $20(22 \%)$ & $34(8 \%)$ \\
\hline Orçamento de capital & & & & $2(2 \%)$ & & & $3(3 \%)$ & $5(1 \%)$ \\
\hline $\begin{array}{l}\text { Mensuração e avaliação de } \\
\text { desempenho }\end{array}$ & $6(15 \%)$ & & & $17(19 \%)$ & $2(4,5 \%)$ & $17(14 \%)$ & $13(15 \%)$ & $55(13 \%)$ \\
\hline Controle organizacional & $3(7,5 \%)$ & $2(13 \%)$ & $2(11 \%)$ & $5(5,5 \%)$ & $6(14 \%)$ & $3(2 \%)$ & & $21(5 \%)$ \\
\hline Controle internacional & & $1(7 \%)$ & & $1(1 \%)$ & & $1(1 \%)$ & & $3(1 \%)$ \\
\hline Múltiplos & & & & $2(2 \%)$ & & $4(3 \%)$ & $3(3 \%)$ & $9(2 \%)$ \\
\hline Sub-total & $11(27,5 \%)$ & $3(20 \%)$ & $2(11 \%)$ & $32(35 \%)$ & $10(23 \%)$ & $30(24 \%)$ & $39(43 \%)$ & $127(30 \%)$ \\
\hline \multicolumn{9}{|l|}{ Outros } \\
\hline $\begin{array}{l}\text { Sistema de informação contábil - } \\
\text { SIC }\end{array}$ & $5(12,5 \%)$ & $2(13 \%)$ & & $14(16 \%)$ & & $1(1 \%)$ & $4(4,5 \%)$ & $26(6 \%)$ \\
\hline Benchmarking & & $3(20 \%)$ & & & & & $1(1 \%)$ & $4(1 \%)$ \\
\hline Qualidade (TQM) & $4(10 \%)$ & $2(13 \%)$ & $3(16,5 \%)$ & $3(3 \%)$ & $6(14 \%)$ & $7(6 \%)$ & & $25(6 \%)$ \\
\hline Just-in-time (JIT) e TOC & & & & $1(2 \%)$ & & $1(1 \%)$ & & $2(0,5 \%)$ \\
\hline Ensino em gerencial & & & & & $3(7 \%)$ & $1(1 \%)$ & & $4(1 \%)$ \\
\hline Gerencial estratégica & $1(2,5 \%)$ & & & & & $3(2 \%)$ & $8(9 \%)$ & $12(3 \%)$ \\
\hline $\begin{array}{l}\text { Preço de transferência e Preço de } \\
\text { venda }\end{array}$ & $2(5 \%)$ & & $7(39 \%)$ & $3(3 \%)$ & & $1(1 \%)$ & $4(4,5 \%)$ & $17(4 \%)$ \\
\hline Estudos sobre CG & $3(7,5 \%)$ & $1(7 \%)$ & $2(11 \%)$ & & $1(2 \%)$ & $21(17 \%)$ & & $28(6,5 \%)$ \\
\hline
\end{tabular}




\begin{tabular}{|l|c|c|c|c|c|c|c|c|}
$\begin{array}{l}\text { Análise de Indicadores } \\
\text { Financeiros }\end{array}$ & $7(17,5 \%)$ & $2(13 \%)$ & & $4(5 \%)$ & $5(11 \%)$ & $6(5 \%)$ & $7(8 \%)$ & $31(7,5 \%)$ \\
\hline Múltiplos & $3(7,5 \%)$ & & $3(16,5 \%)$ & $1(1 \%)$ & $2(4 \%)$ & $22(17 \%)$ & $6(7 \%)$ & $37(8,5 \%)$ \\
\hline Sub-total & $25(62,5 \%)$ & $10(67 \%)$ & $15(83 \%)$ & $26(30 \%)$ & $17(38 \%)$ & $63(50 \%)$ & $30(34 \%)$ & $186(44 \%)$ \\
\hline Total & $\mathbf{4 0}(\mathbf{1 0 0 \%}$ & $\mathbf{1 5}(\mathbf{1 0 0 \% )}$ & $\mathbf{1 8}(\mathbf{1 0 0 \%})$ & $\mathbf{( 1 0 0 \% )}$ & $\begin{array}{c}\mathbf{( 1 0 0 \% )} \\
\mathbf{( 1 0 0 \% )}\end{array}$ & $\mathbf{9 0}(\mathbf{1 0 0 \% )}$ & $\begin{array}{c}\mathbf{4 2 1} \\
(\mathbf{1 0 0} \%)\end{array}$ \\
\hline
\end{tabular}

Fonte: Própria

Conforme Tabela 2, os resultados mostram o tema "outros" com 44\%, planejamento e controle com $30,5 \%$ e custos com $25,5 \%$ das publicações em revistas espanholas de contabilidade.

Se compararmos estes resultados, com pesquisas internacionais como a de Hesford et al. (2007), realizada em revistas de língua inglesa mostra certa sazonalidade das publicações. No período de 1981 a 1990 o tema custos representava aproximadamente $17 \%$ das publicações e controle 74\%. Já no período seguinte (1991 a 2000), o tema custos representava quase $21 \%$ e controle $68 \%$. A grande diferença entre as publicações de língua inglesa e espanhola esta relacionado a centralidade dos temas, as revistas inglesas publicam trabalhos centrados em custos $(20 \%)$ e planejamento e controle (70\%), que são os temas centrais em estudos de contabilidade gerencial, enquanto que o tema outros representa aproximadamente $10 \%$ no período de 1981 a 2000.

\subsection{Seleção da Amostra}

Para a segunda etapa selecionam-se apenas as cinco revistas que apresentam publicações em MAD, conforme Tabela 2, sendo então a amostra objeto de estudo formada por 55 artigos.

Os resultados da amostra mostram certa sazonalidade e uma diminuição gradativa das publicações em MAD no período. Conforme Gráfico 1, pode-se observar que o ano de maior publicação foi 2004 com 8 artigos.

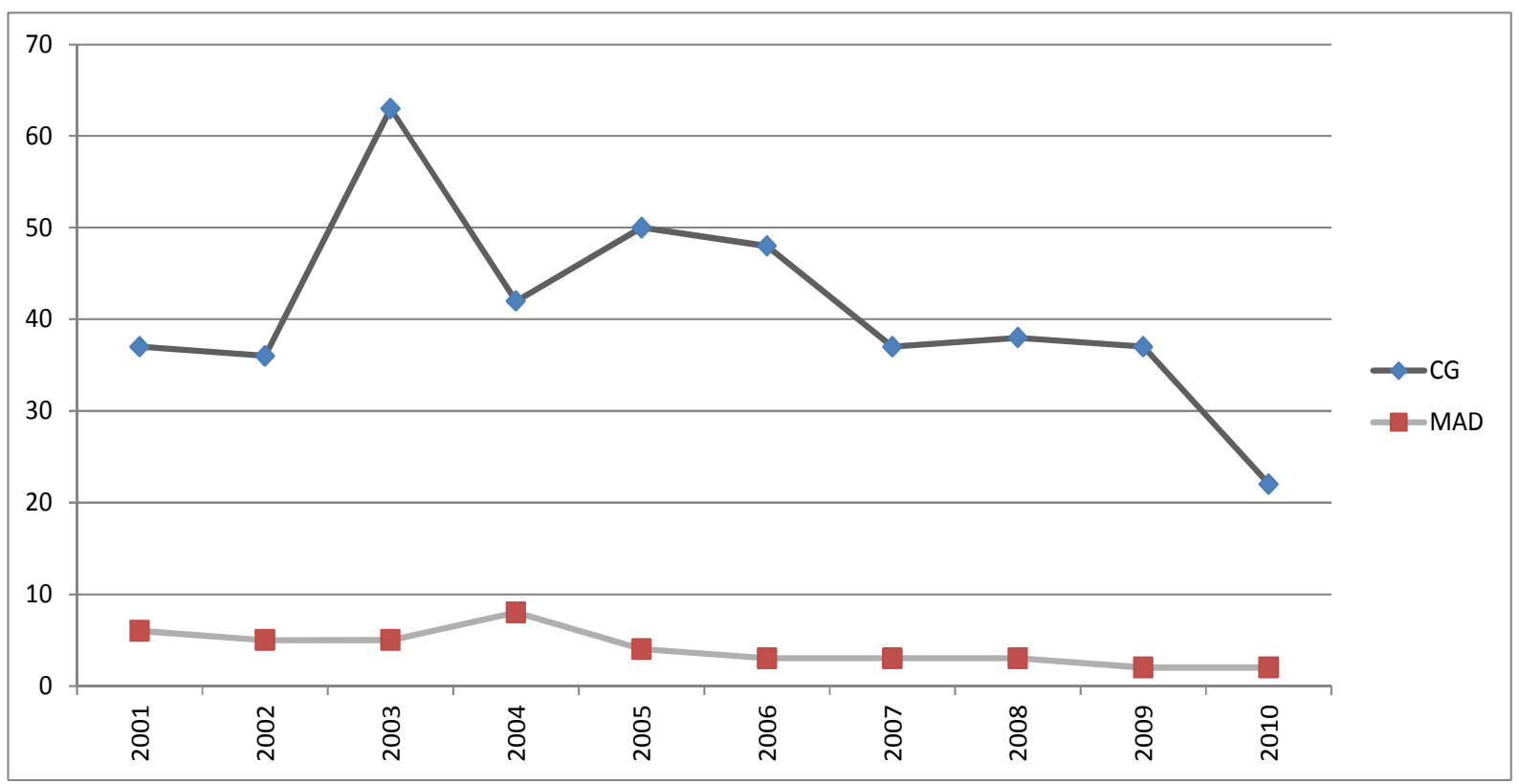

Gráfico 1 - Número de publicações em CG e MAD no período 2001 a 2010.

Fonte: Própria

De uma forma geral, esta ocorrendo uma diminuição das publicações em contabilidade gerencial e também no tema MAD, que representa $13 \%$ do total dos artigos. Se compararmos 
estes resultados, com outras pesquisas como a de Hesford et al. (2007), realizada em revistas de língua inglesa no período de 1981 a 2000, o tema MAD representava 10,9\% em 1981 a 1990 e de 19\% de 1991 a 2000 das publicações em contabilidade gerencial. Para a sequência do trabalho são analisados os 55 trabalhos de MAD.

\section{APRESENTAÇÃO E ANÁLISE DOS RESULTADOS}

\subsection{Classificação dos Artigos}

Baseado em trabalhos de Brown e Gardner (1985), Brown, Gardner e Vasarhelyi (1987), Shields (1997), Perez, Gallardo e Pena (2005), Schekaiban e Ripoll (2005) e Hesford et al. (2007) classificam-se os artigos primeiramente em temas e métodos de pesquisa.

\subsubsection{Temas}

Como ponto de partida para a classificação dos artigos de MAD é utilizada a divisão da literatura entre, (i) estudos sobre medidas de desempenho e sua utilização no gerenciamento das empresas (sem a aplicação de ferramenta específica), (ii) com aplicação da ferramenta específica denominada de Cuadro de Mando Integral ou Balanced Scorecard, (iii) e a determinação de sistemas de incentivos com utilização das medidas de desempenho, (iv) além de um quarto nível denominado de Cuadro de Mando ou Tableau de Bord. Este item busca verificar se no período ocorreu uma mudança entre a utilização da ferramenta Cuadro de Mando adaptada da literatura de origem francesa, para o Cuadro de Mando Integral, de origem norte-americana. A divisão dos temas em subdivisões também levou em consideração os principais tópicos abordados em obras editadas sobre CMI (KAPLAN e NORTON, 1992, 1996, 2000, 2004, 2006).

Assim, o tema foi subdividido em estudos sobre medidas de desempenho que envolve pesquisas relacionados a seleção e aplicação de medidas (indicadores e metas) de performance, seja em nível estratégico, tático ou operacional.

O tópico CMI envolve estudos sobre MAD com aplicação do Cuadro de Mando Integral ou Balanced Scorecard. Este tema foi subdividido em Elaboração do CMI ou BSC, que envolve a construção e desenvolvimento de modelos para aplicação nas organizações. Já a Aplicação Prática em Organização é a descrição de aplicação prática do modelo em uma organização específica. Os estudos de CMI ou BSC referem-se aos trabalhos que visam conhecer aos estágios e os resultados obtidos com a aplicação do instrumento nas organizações. E por fim, sub tópicos integração com o orçamento e a interação com o Custeio Baseado em Atividades - ABC, que envolvem estudos relacionados com a interação e alinhamento destas ferramentas, seja com o orçamento ou o $\mathrm{ABC}$. Os trabalhos com múltiplas abordagens são aqueles estudos que envolvem mais de um tema.

Os trabalhos em Cuadro de Mando envolvem estudos com aplicação prática do instrumento, e também trabalhos de comparação com o Cuadro de Mando Integral.

Finalmente, o sistema de incentivos envolvem estudos com a aplicação de sistema de remuneração variável, utilizando medidas de desempenho.

Os resultados, conforme Tabela 3, mostram que os itens específicos mensuração de desempenho com $25 \%$ e elaboração do CMI com $18 \%$, além de aplicação prática e estudos em CMI com 16,5\% cada, dominam as publicações em MAD. 
Tabela 3 Resultados da classificação das publicações segundo o tema

\begin{tabular}{|c|c|c|c|c|c|c|}
\hline Temas de Pesquisa & REFC & RCAE & RTC & RICG & RHDFC & Total \\
\hline \multicolumn{7}{|l|}{$\begin{array}{l}\text { Medidas de Desempenho } \\
\text { - MD }\end{array}$} \\
\hline $\begin{array}{l}\text { Estudos sobre Medidas de } \\
\text { Desempenho }\end{array}$ & $2(33 \%)$ & $7(41 \%)$ & & $3(17,5 \%)$ & $2(15,5 \%)$ & $14(25 \%)$ \\
\hline \multicolumn{7}{|l|}{$\begin{array}{l}\text { Cuadro de Mando } \\
\text { Integral - CMI }\end{array}$} \\
\hline $\begin{array}{l}\text { Elaboração do CMI ou } \\
\text { BSC }\end{array}$ & & $1(6 \%)$ & $1(50 \%)$ & $3(17,5 \%)$ & $5(38,5 \%)$ & $10(18 \%)$ \\
\hline $\begin{array}{l}\text { Aplicação Prática em } \\
\text { Organização }\end{array}$ & $2(33 \%)$ & $1(6 \%)$ & & $3(17,5 \%)$ & $3(23 \%)$ & $9(16,5 \%)$ \\
\hline Estudos de CMI ou BSC & $1(17 \%)$ & $2(11,5 \%)$ & & $5(29,5 \%)$ & $1(7,5 \%)$ & $9(16,5 \%)$ \\
\hline Integração Orçamento & & $1(6 \%)$ & & & & $1(2 \%)$ \\
\hline Interação com $\mathrm{ABC}$ & & & & $1(6 \%)$ & & $1(2 \%)$ \\
\hline Múltiplos & & $2(11,5 \%)$ & & $2(12 \%)$ & $2(15,5 \%)$ & $6(11 \%)$ \\
\hline \multicolumn{7}{|l|}{ Cuadro de Mando - CM } \\
\hline $\begin{array}{l}\text { Estudos e Aplicações } \\
\text { Práticas }\end{array}$ & $1(17 \%)$ & $1(6 \%)$ & & & & $2(3,5 \%)$ \\
\hline $\mathrm{CM} \times \mathrm{CMI}$ & & & $1(50 \%)$ & & & $1(2 \%)$ \\
\hline \multicolumn{7}{|l|}{$\begin{array}{l}\text { Sistema de Incentivos - } \\
\text { SI }\end{array}$} \\
\hline $\begin{array}{l}\text { Sistema de Remuneração } \\
\text { Variável }\end{array}$ & & $2(12 \%)$ & & & & $2(3,5 \%)$ \\
\hline Total & $6(100 \%)$ & $17(100 \%)$ & $2(100 \%)$ & $17(100 \%)$ & $13(100 \%)$ & $55(100 \%)$ \\
\hline
\end{tabular}

Fonte: Própria

\subsubsection{Métodos}

Para a classificação dos artigos foi utilizada a abordagem de Birnberg, Shields e Young (1990) e Hesford et al. (2007), que dividem os métodos em nove categorias, descritas no Quadro 2.

Quadro 2 Classificação e definição dos métodos de pesquisa

\begin{tabular}{|l|l|}
\hline Métodos de pesquisa & Definição \\
\hline Analítica & $\begin{array}{l}\text { Envolve o estudo e avaliação aprofundados de informações } \\
\text { disponíveis na tentativa de explicar o contexto de um fenômeno. }\end{array}$ \\
\hline Arquivo/documental & $\begin{array}{l}\text { Envolve a utilização de informações que ainda não tiveram um tratamento } \\
\text { cientifico. }\end{array}$ \\
\hline Caso & $\begin{array}{l}\text { Envolve a investigação de fenômenos, inclusive pessoas, procedimentos e } \\
\text { estruturas de um processo, departamento ou de uma organização. }\end{array}$ \\
\hline Experimental & $\begin{array}{l}\text { Envolve a manipulação e tratamentos na tentativa de estabelecer relações de } \\
\text { causa-efeito nas variáveis investigadas. }\end{array}$ \\
\hline Campo & Envolve a investigação de fenômenos, inclusive pessoas, procedimentos e \\
\hline
\end{tabular}




\begin{tabular}{|l|l|}
\hline & estruturas, unidades e atividades em organizações. \\
\hline Framework & Envolve o desenvolvimento de um novo conceito, de novas perspectivas. \\
\hline Revisão & Envolve basicamente síntese e revisão de literatura já conhecida. \\
\hline Levantamento & $\begin{array}{l}\text { Envolve a investigação de um grupo de pessoas, geralmente realizado via a } \\
\text { aplicação de questionário. }\end{array}$ \\
\hline Outros & Não se enquadra em nenhum dos outros métodos. \\
\hline
\end{tabular}

Fonte: Baseado em Birnberg, Shields e Young (1990) e Hesford et al. (2007).

De acordo com a Tabela 4, a classificação dos artigos mostra uma predominância dos métodos de revisão e estudo de caso, além do levantamento e dos estudos de campo. Já os métodos analíticos, arquivo/documental e framework não são aplicados nas pesquisas em MAD nas revistas espanholas.

Tabela 4 Resultados da classificação dos artigos segundo o método de pesquisa

\begin{tabular}{|l|c|c|}
\hline Métodos de pesquisa & Artigos (2001-2010) & Percentual (\%) \\
\hline Analítica & & 27 \\
\hline Arquivo/documental & 15 & 2 \\
\hline Caso & 1 & 11 \\
\hline Experimental & 6 & 47 \\
\hline Campo & & 13 \\
\hline Framework & 26 & \\
\hline Revisão & 7 & $\mathbf{1 0 0}$ \\
\hline Levantamento & & $\mathbf{2 5}$ \\
\hline Outros & $\mathbf{5 5}$ & \\
\hline Total & & \\
\hline Fonte: Proprâ & & \\
\hline
\end{tabular}

Fonte: Própria

Os resultados demonstram uma predominância pela investigação de literatura já conhecida, bem como de fenômenos, procedimentos e estruturas em uma única organização. Ao comparar os resultados com os obtidos por Hesford et al. (2007), encontra-se algumas diferenças sensíveis, as revistas de língua inglesa procuram publicar artigos que envolvem o desenvolvimento de novos conceitos e perspectivas (framework), além da avaliação aprofundada das informações disponíveis na tentativa de explicar o contexto de um fenômeno (analítico).

Para aprofundar o estudo do perfil das publicações em MAD procede-se a seguir a análise das características da revista, dos artigos, dos autores e das citações.

\subsection{Características das Revistas}

A análise das características é uma forma de compreender as linhas e tendências editoriais da revista. Conforme Tabela 5, os resultados mostram que 65,5\% dos artigos têm foco no Cuadro de Mando Integral (CMI), enquanto 25\% em medidas de desempenho (MD), $6 \%$ em cuadro de mando $(\mathrm{CM})$ e $3,5 \%$ em sistema de incentivos (SI). 
Tabela 5 Classificação dos artigos segundo revista e temas

\begin{tabular}{|c|c|c|c|c|c|}
\hline Revistas & CMI & SI & MD & CM & Total \\
\hline REFC & $3(5,5 \%)$ & & $2(3,5 \%)$ & $1(2 \%)$ & $\mathbf{6}(\mathbf{1 1 \%})$ \\
\hline RCAE & $7(12,5 \%)$ & $2(3,5 \%)$ & $7(12,5 \%)$ & $1(2 \%)$ & $\mathbf{1 7}(\mathbf{3 1 \%})$ \\
\hline RTC & $1(2 \%)$ & & & $1(2 \%)$ & $\mathbf{2}(\mathbf{3 , 5 \%})$ \\
\hline RICG & $14(25,5 \%)$ & & $3(5,5 \%)$ & & $\mathbf{1 7 ( 3 1 \% )}$ \\
\hline RHDFC & $11(20 \%)$ & & $2(3,5 \%)$ & & $\mathbf{1 3}(\mathbf{2 3 , 5 \%})$ \\
\hline Total & $\mathbf{3 6}(\mathbf{6 5 , 5 \% )}$ & $\mathbf{2 ( 3 , 5 \% )}$ & $\mathbf{1 4}(\mathbf{2 5 \%})$ & $\mathbf{3 ( 6 \% )}$ & $\mathbf{5 5}(\mathbf{1 0 0 \% )}$ \\
\hline
\end{tabular}

Fonte: Própria

Quanto à metodologia aplicada nas pesquisas, a REFC apresenta todos os trabalhos voltados a aplicação prática, $50 \%$ e $17 \%$ em estudo de caso e de campo e $33 \%$ em levantamento. A RCAE apresenta trabalhos focados no método de revisão com 53\%, 17,5\% em estudo de caso, além de $12 \%$ em estudo de campo e levantamento. A RTC tem todas as publicações com aplicação do método de revisão, já a RICG tem 35,5\% em revisão, 17,5\% em estudo de caso e levantamento, respectivamente. Os métodos mais utilizados na RHDFC são a revisão com $69 \%$ e o estudo de caso com $31 \%$. Os métodos analíticos e framework não são objeto de aplicação nos estudos de MAD, conforme Tabela 6.

Tabela 6 Classificação dos artigos segundo revista e método

\begin{tabular}{|c|c|c|c|c|c|c|}
\hline Revistas & Caso & Experimental & Campo & Revisão & Levantamento & Total \\
\hline REFC & $3(50 \%)$ & & $1(17 \%)$ & & $2(33 \%)$ & $6(100 \%)$ \\
\hline RCAE & $3(17,5 \%)$ & $1(5,5 \%)$ & $2(12 \%)$ & $9(53 \%)$ & $2(12 \%)$ & $17(100 \%)$ \\
\hline RTC & & & & $2(100 \%)$ & & $2(100 \%)$ \\
\hline RICG & $5(29,5 \%)$ & & $3(17,5 \%)$ & $6(35,5 \%)$ & $3(17,5 \%)$ & $17(100 \%)$ \\
\hline RHDFC & $4(31 \%)$ & & & $9(69 \%)$ & & $13(100 \%)$ \\
\hline Total & $15(100 \%)$ & $1(100 \%)$ & $6(100 \%)$ & $26(100 \%)$ & $7(100 \%)$ & $55(100 \%)$ \\
\hline
\end{tabular}

Fonte: Própria

Embora, estes resultados mostram uma grande gama de métodos utilizados, as publicações apresentam certa concentração, e inclusive revista com um único método de pesquisa. Uma particularidade chama a atenção nos resultados, se comparados a pesquisas realizadas em outros países (como a de Hesford et al., 2007), em relação aos métodos empregados, é o número limitado de pesquisas com grupos maiores de pessoas ou organizações, com aplicação de questionário para a coleta das informações. Eles correspondem a apenas $13 \%$ do total, sendo que em pesquisas norte-americanas este percentual chega a ser de aproximadamente $20 \%$ do total das publicações em gerencial.

\subsection{Características dos Artigos}

As pesquisas em CMI são predominantemente desenvolvidas por meio de revisão da literatura (47\%) e estudo de caso (30,5\%), conforme Tabela 7. 
Tabela 7 Classificação dos artigos segundo tema e método

\begin{tabular}{|l|c|c|c|c|c|}
\hline \multicolumn{1}{|c|}{ Métodos } & CMI & SI & MD & CM & Total \\
\hline Caso & $11(30,5 \%)$ & & $2(14,5 \%)$ & $2(67 \%)$ & $\mathbf{1 5}(\mathbf{2 7 , 5 \% )}$ \\
\hline Experimental & & & $1(7 \%)$ & & $\mathbf{1}(\mathbf{2 \%})$ \\
\hline Campo & $5(14 \%)$ & & $1(7 \%)$ & & $\mathbf{6}(\mathbf{1 1 \%})$ \\
\hline Revisão & $17(47 \%)$ & $2(100 \%)$ & $6(43 \%)$ & $1(33 \%)$ & $\mathbf{2 6 ( 4 7 , 5 \% )}$ \\
\hline Levantamento & $3(8,5 \%)$ & & $4(28,5 \%)$ & & $\mathbf{7 ( 1 2 \% )}$ \\
\hline Total & $\mathbf{3 6 ( 1 0 0 \% )}$ & $\mathbf{2 ( 1 0 0 \% )}$ & $\mathbf{1 4}(\mathbf{1 0 0 \% )}$ & $\mathbf{3 ( 1 0 0 \% )}$ & $\mathbf{5 5 ( 1 0 0 \% )}$ \\
\hline
\end{tabular}

Fonte: Própria

Conforme Tabela 7, os trabalhos de medidas de desempenho são desenvolvidos por meio de revisão, levantamento e estudo de caso. Já o tema CM tem a aplicação do método de revisão que envolve o estudo e análise comparativa dos resultados, além de dois estudos aplicados em organizações. Os trabalhos de sistema de incentivos são elaborados mediante a revisão de literatura.

Para verificar o nível de citação dos artigos foi realizada uma consulta no Google Acadêmico (Google scholar), onde constatou-se que apenas 20 artigos foram referenciados em outras publicações. Dois artigos se destacaram com 11 citações cada: "Diseño e implantación del Cuadro de Mando Estratégico: el caso de tres empresas multinacionales" de Luis FernándezRevuelta Pérez e Urban Ask e "Un Análisis de la Flexibilidad del Cuadro de Mando Integral (CMI) en su Adaptación a la Naturaleza de las Organizaciones de Mónica Santos Cebrián e Esther Fidalgo Cerviño.

Estes resultados mostram que os trabalhos não são referenciados em novas pesquisas, das 55 publicações da amostra 20 tiveram citações na rede. Embora, os artigos de 2009 e 2010 ainda não tivessem tempo suficiente para serem citados em novas pesquisas, os de anos anteriores deveriam já ter sido referenciados. Isto mostra de certa forma que não há continuidade nas pesquisas em MAD, dificultando o aperfeiçoamento e melhoria dos temas, com a formação de redes de pesquisa e centros de excelência na área.

Neste ponto, talvez as críticas de Zimmerman (2001) e Ittner e Larcker (2001) façam sentido, para que os estudos tenham continuidade é necessário de forma preditiva aprofundar e avançar em termos de revisão da literatura, para a partir desta aplicar os modelos em organizações para testá-los e na sequência também a verificação da sua eficiência e possíveis vantagens, limitações, além é claro de generalizações que possam consolidar a área de pesquisa. Para tanto, é necessário a descrição clara das etapas do estudo, com aplicação de diferentes métodos de pesquisa. Aliado a isto, em relação à contribuição dos trabalhos verifica-se certa limitação em termos de definição das variáveis como, do objetivo, critério, atributo, fator crítico de sucesso ou ponto de vista fundamental (KEENEY, 1992).

De uma forma geral, estes trabalhos apresentam mapas prontos, sem discutir como foram elaborados, que aspectos foram considerados. Ou seja, foram desenvolvidos com auxilio de especialistas, como e quais critérios e sub critérios foram considerados, quais são os níveis de desempenho desejados, o que ocorrerá se o desempenho não for atingido, sistemas de incentivos relacionados, como as metas serão mensuradas etc. Em parte, estes trabalhos são soluções prontas, pacotes ou fórmulas de remédio, que servem para todos os sintomas ou problemas dos pacientes. Em geral, o desenvolvimento destes modelos induz que somente há uma forma de resolver os problemas de gestão, entretanto, conforme a literatura os sistemas de mensuração e avaliação de desempenho podem ser construídos com aplicação de diferentes abordagens, em contextos singulares, e para atender a diferentes objetivos: controle, tomada e apoio a decisão etc. 


\subsection{Características dos Autores}

Em relação ao número de autores por artigo, $40 \%$ apresentam um único autor por artigo, $45,5 \%$ dois autores, $12,5 \%$ três autores e apenas $2 \%$ quatro autores, conforme Tabela 8 . Não há publicação com 5 ou mais autores por artigo.

Tabela 8 Número de autores por artigo

\begin{tabular}{|c|c|c|c|c|c|c|}
\hline Autores & REFC & RCAE & RTC & RICG & RHDFC & Total \\
\hline $\mathbf{1}$ & $2(33 \%)$ & $6(35 \%)$ & & $8(47 \%)$ & $6(46 \%)$ & $22(40 \%)$ \\
\hline $\mathbf{2}$ & $2(33 \%)$ & $8(47 \%)$ & $(100 \%)$ & $8(47 \%)$ & $5(39 \%)$ & $25(45,5 \%)$ \\
\hline $\mathbf{3}$ & $2(34 \%)$ & $3(18 \%)$ & & $1(6 \%)$ & $1(7,5 \%)$ & $7(12,5 \%)$ \\
\hline $\mathbf{4}$ & & & & & $1(7,5 \%)$ & $1(2 \%)$ \\
\hline $\mathbf{5}$ ou $+\mathbf{5}$ & & & & & & \\
\hline Total & $\mathbf{6 ( 1 0 0 \% )}$ & $\mathbf{1 7}(\mathbf{1 0 0} \%)$ & $\mathbf{2 ( 1 0 0 \% )}$ & $\mathbf{1 7}(\mathbf{1 0 0 \% )}$ & $\mathbf{1 3}(\mathbf{1 0 0 \% )}$ & $\mathbf{5 5}(\mathbf{1 0 0 \% )}$ \\
\hline
\end{tabular}

Fonte: Própria

Ao comparar os resultados da Tabela 8 com pesquisa realizada por Moya e Prior (2008) no período de 1996 a 2005 em periódicos de contabilidade da Espanha, onde artigos com 1 autor representavam em 1996 (41\%), 2 autores (37\%), 3 autores (14,5\%), 4 autores (3\%) e 5 autores $4 \%$. Em decorrência dos resultados, os autores da pesquisa já mostravam certa preocupação com a falta de publicações a partir de grupos de pesquisa e redes sociais.

No entanto, estes resultados são semelhantes aos encontrados em publicações de língua inglesa, onde autores com um artigo representam aproximadamente $42 \%$, dois autores $39 \%$, três $16 \%$ e quatro e mais autores 3\% dos artigos (HESFORD ET AL.,2007).

Conforme Tabela 9, a maioria dos autores (92\%) publicou apenas um artigo na revista e $8 \%$ dois artigos. Não há autores com 3 ou mais publicações na mesma revista. Isto mostra que as revistas não apresentam grande concentração em termos de autores.

Tabela 9 Quantidade de artigos publicados por autor na revista

\begin{tabular}{|c|c|c|c|c|c|c|}
\hline Artigos & REFC & RCAE & RTC & RICG & RHDFC & Total \\
\hline 1 & $12(100 \%)$ & $22(84 \%)$ & $4(100 \%)$ & $23(92 \%)$ & $21(95,5 \%)$ & $82(92 \%)$ \\
\hline 2 & & $4(16 \%)$ & & $2(8 \%)$ & $1(4,5 \%)$ & $7(8 \%)$ \\
\hline 3 ou +3 & & & & & & \\
\hline Total & $\mathbf{1 2}(\mathbf{1 0 0 \% )}$ & $\mathbf{2 5}(\mathbf{1 0 0 \%})$ & $\mathbf{4 ( 1 0 0 \% )}$ & $\mathbf{2 5}(\mathbf{1 0 0 \% )}$ & $\mathbf{2 2 ( 1 0 0 \% )}$ & $\mathbf{8 9}(\mathbf{1 0 0})$ \\
\hline
\end{tabular}

Fonte: Própria

De uma forma geral, considerando todas as revistas, quatro autores (Muñiz González, L.; Giner Fillol, A.; Ripoll Feliu, V.; Martínez Ramos, M.) apresentam três publicações. Analisando os resultados, percebe que não há um autor predominante nas publicações de MAD nas revistas espanholas. Isto se deve em parte porque na Espanha poucos autores têm foco exclusivo em um tema.

\subsection{Características das Citações}

A identificação e análise das citações das publicações é uma forma de entender as bases referenciais do tema MAD. Assim, foram identificadas 1.336 citações referentes aos 55 
artigos da amostra, média de aproximadamente 24 referências por artigo. Conforme Tabela 10, Kaplan, R.S. com 85, aproximadamente $6 \%$ do total das referencias é o autor com maior número de citações. Deste total de citações, 62 são em conjunto com Norton, D., isto demonstra o sucesso da ferramenta CMI.

Tabela 10 Número de citações por autor

\begin{tabular}{|l|c|l|c|}
\hline \multicolumn{1}{|c|}{ Autores } & Número de Citações & \multicolumn{1}{c|}{ Autores } & Número de Citações \\
\hline Kaplan, R.S. & 85 & Cooper, R. & 11 \\
\hline Norton, D. & 62 & Hopwood, A.G. & 10 \\
\hline AECA & 25 & Chenhal, R.H. & 9 \\
\hline Ittner, C.D. & 21 & Simons, R. & 9 \\
\hline Larcker, D.F. & 16 & Amat Salas, O. & 7 \\
\hline Scapens, R.W. & 12 & Johnson, H.T. & 7 \\
\hline Othey, D. & 11 & Ripoll Feliu, V. & 8 \\
\hline
\end{tabular}

Fonte: Própria

Já a AECA (Asociación Española de Contabilidad y Administración de Empresas) foi a terceira referencia mais utilizada com 25 citações. A utilização das obras editadas pela AECA é uma particularidade das publicações espanholas de contabilidade. Entre os autores espanhóis destaque para Oriol Amat Salas e Vicente M. Ripoll Feliu, com 8 e 7 citações cada. Os resultados indicam forte influência de citações norte-americanas, consequiência em parte do grande sucesso que as ferramentas de MAD, principalmente o CMI ou BSC, obtiveram na literatura e na prática das organizações. Mas também pela carência de centros de pesquisa e principalmente de pesquisadores em contabilidade gerencial. Na Espanha, assim como em outros países, os doutorandos e consequentemente a formação de novos doutores que se dediquem a pesquisar em contabilidade gerencial é cada vez menor.

\section{CONCLUSÕES E RECOMEDAÇÕES}

Os resultados da pesquisa demonstram que a contabilidade gerencial ainda não ocupa o lugar de destaque nas revistas de contabilidade na Espanha. Isto também se aplica ao tema mensuração e avaliação de desempenho, que vem perdendo força em relação ao número de publicações.

Quanto aos temas de MAD pesquisados forte predominância do tópico CMI, se comparados a medidas de desempenho, Cuadro de Mando e sistema de incentivos, com aplicação dos métodos de revisão e estudo de caso e de campo. Se comparar esses resultados com os de outros países, como a de Hesford et al. (2007) realizada nos EUA, nas revistas espanholas são limitadas as publicações de MAD com aplicação do estudo de campo e de levantamento. Este resultado mostra que há um potencial muito grande na realização de estudos com a aplicação dos métodos de estudo de campo e de levantamento em futuras pesquisas na Espanha. Com esta ampliação do campo de estudo talvez se possa fazer algumas generalizações, com a consolidação de aspectos imprescindíveis ao desenvolvimento e aplicação dos modelos de gestão.

Quanto aos métodos de estudos aplicados (revisão e estudo de caso), uma explicação para este fenômeno é descrito por Atkinson et al. (1997), ao fazer referencia que os autores europeus 
aplicam em grande parte teorias advindas da sociologia e psicologia e estudam o papel da contabilidade gerencial nas organizações. Já as publicações nos Estados Unidos, são baseadas nas teorias econômicas e estudos com aplicação de métodos experimentais e forte predomínio de métodos estatísticos.

Entre os principais resultados da pesquisa pode-se destacar a baixa citação das publicações, dos 55 artigos da amostra 20 tiveram citações na rede. Isto em parte pela influencia da literatura norte-americana, mas também pela falta de continuidade das pesquisas, o que reflete a pouca incidência de pesquisadores centrados no tema.

Entre os estudos publicados no período, $40 \%$ deles apresenta apenas um autor, o que pode ser outro forte indício da falta de pesquisas conjuntas e resultantes de grupos de pesquisa e redes sociais formados por pesquisadores de diferentes países e culturas.

Um aspecto que merece destaque é a mudança da influencia francesa por meio do Cuadro de Mando ou Tableau de Bord para o predomínio do Cuadro de Mando Integral ou Balanced Scorecard de origem norte-americana. Em 2002 há uma aplicação prática do CM em uma empresa, já o trabalho de 2006 mescla o CM com o CMI, gerando uma proposta para aplicação prática em organizações. O trabalho de 2005 mostra as principais diferenças em o CM e o CMI. Isto indica que no período houve uma mudança na configuração das abordagens em MAD, de uma influencia da literatura francesa através do Tableau de Bord, para uma predominantemente norte-americana por meio do Balanced Scorecard.

Alguns trabalhos trazem contribuições modestas a literatura, isto porque não apresentam de forma estrutura qual é o objetivo, critério, atributo, fator crítico de sucesso ou ponto de vista fundamental utilizado no desenvolvimento do modelo. Aliando a isto, não definem como foi realizada a escolha dos objetivos, indicadores e metas e como foram construídas as escalas, quais resultados são aceitáveis ou não aceitáveis e quem são os responsáveis para atingir os resultados esperados. Isto sem mencionar outros aspectos: como os resultados serão mensurados e quem será o responsável pela implementação e controle, como será a melhoria contínua do sistema etc.

Entre as recomendações para futuros trabalhos está a possibilidade de ampliar a pesquisa com inclusão de outras revistas espanholas e de países de língua portuguesa, e explorar individualmente os pontos fortes e as oportunidades para cada um dos temas.

\section{REFERENCIAS}

ABERNETHY, M. A.; HORNE, M. et al. A multi-method approach to building causal performance maps from expert knowledge. Management Accounting Research 16(2). 2005, p.135-155.

ALIGULIYEV, R. M. Performance evaluation of density-based clustering methods. Information Sciences 179(20). 2009, p. 3583-3602.

ATKINSON, A. A.; et al. Contabilidade gerencial. São Paulo: Atlas, 2000.

AZOFRA, V.; PRIETO, B.; ALCIA, S. The usefulness of a performance measurement system in the daily life of an organisation: a note on a case study. The British Accounting Review 35(4), 2003, p. 367-384.

BAIMAN, S. Agency research in managerial accounting: a survey. Journal of Accounting Literature, Vol.1, 1982. p.154-213. 
BALASUBRAMANIAN, S.; GUPTA, M. Structural metrics for goal based business process design and evaluation. Business Process Management Journal 11(6), 2005, P. 680-694.

BALLVÉ, A. M.; AMAT SALAS, O. Los Cuadros de Mando como Sistemas Interactivos. Revista Iberoamericana de Contabilidad de Gestión. Vol. IV (8). 2006, p.1-16.

BARBER, N.; SCARCELLI, J. M. Enhancing the assessment of tangible service quality through the creation of a cleanliness measurement scale. Managing Service Quality 20(1). 2010, p. 70-88.

BIRNBERG, J. G.; SHIELDS, M. D.; YOUNG, S. M. The case for multiple methods in empirical management accounting research (with an illustration from budget setting). Journal of Management Accounting Research, Vol. 2,1990, p. 33-66.

BLANCO, F. El control integrado de gestión. México: Limusa, 1976. p. 93-156.

BROMWICH, M. The case for strategic management accounting: the role of accounting information for strategy in competitive markets. Accounting, Organizations and Society, 15(1), 1990, p.27-46.

BROWN, L. D. GARDNER, J. C. Applying citation analysis to evaluate the research contributions of accounting faculty and doctoral programs. The Accounting Review, 1985, pp. $262-277$.

BROWN, L. D. GARDNER, J. C. Using citation analysis to assess the impact of journals and articles on contemporary accounting research (CAR). Journal of Accounting Research, 1985, p. 84-109.

BROWN, L. D., GARDNER, J. C. VASARHELYI, M. A. An analysis of the research contributions of Accounting, Organizations and Society, 1976-1984. Accounting, Organizations and Society, 1987, pp.193-204.

BUYTENDIJK, F.; HATCH, T.; PIETRO, M. Scenario-based strategy maps. Business Horizons In Press, Corrected Proof, 2010.

CARL, O.; CHRIS, D. Packaging scorecard - a packaging performance evaluation method. Packaging Technology and Science 16(1). 2003, P. 9-14.

CHEN, M.Y.; HUANG, M.J.; CHENG, Y.C. Measuring knowledge management performance using a competitive perspective: An empirical study. Expert Systems with Applications 36(4). 2009, p.8449-8459.

CHIN, K.S.; LO, K.C.; et al. Development of user-satisfaction-based knowledge management performance measurement system with evidential reasoning approach. Expert Systems with Applications 37(1): 2010, p.366-382.

COOPER, D. J. Tidiness, muddle, and things: commonalities and divergencies in two approaches to management accounting research. Accounting, Organizations and Society, 8(2/3), 1983, p.269-286. 
COOPER, R. Does your company need a new cost system?. Journal of Cost Management, 1(1), 1987, p.45-49.

COVALESKI , M. A.; DIRSMITH, M. W.; SAMUEL, S. Managerial accounting research: the contributions of organizational and sociological theories. Journal of Management Accounting Research, 8, 1996, p.1-35.

DENT, J. F. Strategy, organization, and control: some possibilities for accounting research. Accounting, Organizations and Society, 15(1/2), 1990, p.3-25.

DEY, P. K. Integrated project evaluation and selection using multiple-attribute decisionmaking technique. International Journal of Production Economics 103(1). 2006, P. 90-103.

ENSSLIN, L; ENSSLIN, S.R. Revisão Sistêmica da Literatura. Material didático apresentado na Disciplina de Avaliação de Desempenho do Programa de Pós-Graduação em Engenharia de Produção da Universidade Federal de Santa Catarina, primeiro trimestre, 2010.

ENSSLIN, L.; GIFFHORN, E.; ENSSLIN, S. R.; PETRI, S. M.; VIANNA, W. B. Avaliação do Desempenho de Empresas Terceirizadas com o Uso da Metodologia Multicritério de Apoio à Decisão - Construtivista. Revista Pesquisa Operacional, (2010) In press.

ENSSLIN, L.; MONTIBELLER, G.N.; e NORONHA, S.M. Apoio à decisão: metodologia para estruturação de problemas e avaliação multicritério de alternativas. Florianópolis: Insular, 2001.

FERNÁNDEZ, A. Les nouveaux tableaux de bord des décideurs. Paris: Éditions Organisation. 2000.

HESFORD et al. Management accounting: a bibliographic study. In: CHAPMAN, Christopher; HOPWOOD, Anthony G.; SHIELDS, Michael (Org.). Handbook of management accounting research. Amsterdam: Elsevier, 2007. p. 3-26. v.1. cap. 1.

HIROMOTO, T. Another hidden edge: Japanese management accounting. Harvard Business Review, 66(4), 1988, p.22-25.

HOPWOOD, A. G. Towards an organizational perspective for the study of accounting and information systems. Accounting, Organizations and Society, 3(1), 1978, p. 3-13.

HSU, Y.L., LI, W.C. et al. Structuring critical success factors of airline safety management system using a hybrid model. Transportation Research Part E: Logistics and Transportation Review 46(2). 2009, p. 222-235.

HU, H.Y., LEE, Y.C. et al. Using BPNN and DEMATEL to modify importance-performance analysis model - A study of the computer industry. Expert Systems with Applications 36(6): 2009, P.9969-9979.

HSU, Y.L., LI, W.C.; CHEN, K.W. (2009), Structuring critical success factors of airline safety management system using a hybrid model. Transportation Research Part E: Logistics 
and Transportation Review 46(2): 222-235.

ITTNER, C. D.; LARCKER, D. F. Assessing empirical research in managerial accounting: a value-based management perspective. Journal of Accounting and Economics, v. 32, p. 349410, 2001.

JANSSEN, R., H. GOOSEM, et al. Decision support for integrated wetland management. Environmental Modelling \& Software 20(2). 2005, P. 215-229.

JOHNSON, H. T.; KAPLAN, R. S. Relevance lost: the rise and fall of management accounting. Boston, MA: Harvard Business School Press, 1987.

KAPLAN, R. S.; NORTON, D, P. Alinhamento: usando o Balanced Scorecard para criar sinergias corporativas. Rio de Janeiro: Elsevier, 2006.

. Mapas estratégicos: convertendo a2010tivos intangíveis em resultados tangíveis. Rio de Janeiro: Campus, 2004.

Organização orientada para a estratégia. Rio de Janeiro: Campus, 2001.

Estratégia em Ação - Balanced Scorecard, $7^{\mathrm{a}}$. Ed. Rio de Janeiro: Campus, 1997.

.The balanced scorecard: measures that drive performance. Harvard Business Review, 70(1), 1992, p.71-79.

KAPLAN, R. S. Measuring manufacturing performance: a new challenge for management accounting research. The Accounting Review, 58(4), 1983, p.686-705.

KEENEY, R.L. Value focused-thinking: a path to creative decision-making. Cabridge: Harvard Univ. Press, 1992.

LI, Y.; LIAO, X. Decision support for risk analysis on dynamic alliance. Decision Support Systems 42(4). 2007, p. 2043-2059.

LACERDA, R., ENSSLIN, L., ENSSLIN,S.R. Gerenciamento de Portfólio e Avaliação de Desempenho: Um estudo de caso sobre apoio à decisão. Mundo Project Management. Ano 5, número 29, out/Nov, pp. 60-69. 2009.

LUNKES, R. J.; RIPOLL, V. M.; ROSA, F. S. Redes Sociais e Internacionalização da Contabilidade Gerencial: Um Estudo em Publicações de Língua Espanhola. XIII Congresso de Contabilidade e Auditoria. Porto (Portugal), 2011.

MACINTOSH, N. B.; SCAPENS, R. W. Structuration theory in management accounting. Accounting, Organizations and Society, 15(5), 1990, p.455-477.

MEYER, J. Le contrôle de gestion. Paris: Presses Universitaires de France, 1969.

MOHAMADABADI, H. S.; TICHKOWSKY, G.; KUMAR, A. Development of a multicriteria assessment model for ranking of renewable and non-renewable transportation fuel vehicles. Energy, v. 34, n. 1, p. 112-125, 2009. 
MOYA, S. ¿Quién Publica en las Revistas Españolas de Contabilidad? Análisis bibliométrico del periodo 1996-2005. Revista Española de Financiación y Contabilidad, Vol. XXXIV, No.138, 2008, p.353-374.

PEREZ, B. E.; GALLARDO, A. L.; PEÑA, C. R. La investigación empirica en contabilidad de gestión en España: análisis de las publicaciones españolas. Revista Española de Financiación y Contabilidad, Vol. XXXIV, No.124, Enero - Marzo, 2005, p.183-210.

ROSA, F. S., ENSSLIN, S. R., ENSSLIN, L. Evidenciação Ambiental: Processo Estruturado de Revisão de Literatura sobre Avaliação de Desempenho da Evidenciação Ambiental. Sociedade, Contabilidade e Gestão (UFRJ). , v.4, p.4 - 8, 2009.

ROSA, F. S., FERREIRA, A. C. S., ENSSLIN, S. R., ENSSLIN, L. Evidenciação Ambiental: Contribuição da Metodologia Multicritério para Identificação dos Aspectos Financeiros para a Gestão Ambiental. Contabilidade Vista \& Revista. , v.21, p.1 - , 2010

SCHEKAIBAN, N.; RIPOLL, V. M. Estudio comparativo sobre investigación en Contabilidad de Gestión: Espana-México, 2005.

SELDEN, S.; SOWA, C. J. E. Testing a Multi-Dimensional Model of Organizational Performance: Prospects and Problems. J. Public Adm. Res. Theory. 14(3): 2004, p.395-416.

SHANK, J.; GOVINDARAJAN, V. Strategic cost management: the new tool for competitive advantage. New York, NY: The Free Press, 1993.

SHIELDS, M. D. Research in management accounting by North Americans in the 1990s. Journal of Management Accounting Research, 1997, p.3-61.

SIMONS, R. Levers of Control - How Managers Use Innovative Control Systems to Drive Strategic Renewal. Boston: Harvard Business, 1995.

WOUTERS, M.; WILDEROM, C. Developing performance-measurement systems as enabling formalization: A longitudinal field study of a logistics department. Accounting, Organizations and Society 33(4-5). 2008, p.488-516.

YOUNG, S. M.; SELTO, F. H. New manufacturing practices and cost management: a review of the literature and directions for future research. Journal of Accounting Literature, 10, 1991, p.265-298.

ZIMMERMAN, J. L. Conjectures regarding empirical managerial accounting research. Journal of Accounting and Economics, v. 32, 2001, p. 411-427. 